\title{
Homenaje
}

\section{Jorge A. Flores Ochoa, una vasta obra antropológica hecha desde y para el sur andino peruano}

\section{Guillermo Salas Carreño}

(iD) https://orcid.org/0000-0003-4770-4550

Pontificia Universidad Católica del Perú guillermo.salas@pucp.pe

RESUMEN

El 20 de agosto de 2020 falleció el doctor Jorge A. Flores Ochoa, una figura fundamental en la antropología cuzqueña y peruana por sus múltiples méritos como investigador, docente, intelectual comprometido y animador cultural infatigable. Este texto repasa sus principales temas de investigación e interés, en particular sus notables contribuciones y constante estudio de las sociedades de pastores de camélidos en los Andes y, asociado a esto, sus estudios sobre estrategias de manejo de la complejidad agroecológica andina. También se presta atención a sus investigaciones sobre cultura material y arte cuzqueño colonial y republicano, su etnografía de las celebraciones religiosas de la ciudad y la región, y sus contribuciones respecto de la antropología de la ciudad del Cuzco y sus interpretaciones y análisis sobre la historia e importancia del incanismo cuzqueño contemporáneo.

Palabras clave: antropología peruana, pastores de camélidos, fiesta religiosa, arte colonial, incanismo, Puno, Cuzco. 


\title{
Jorge Flores Ochoa, a vast anthropological work made from and for the Southern Peruvian Andes
}

\author{
ABSTRACT
}

Recently, on August 20, 2020, Dr. Jorge A. Flores Ochoa passed away. He has been a fundamental figure in Cuzco and Peruvian anthropology due to his multiple merits as a researcher, professor, engaged intellectual and tireless cultural animator. This text reviews his main research themes and interests, in particular his notable contributions and constant study of Andean pastoralist societies and, associated with this, his studies regarding the management of Andean agro-ecological complexity. The article then moves to his research on Cuzco's colonial and republican art and material culture, his ethnography of the city's and region's religious celebrations, his broader contributions to the anthropology of the city of Cuzco and his interpretations and analysis on the history and importance of contemporary Cuzco Incanism.

Keywords: Peruvian anthropology, camelid pastoralists, religious celebrations, colonial art, inkanism, Puno. Cuzco. 
El 20 de agosto de 2020 falleció el doctor Jorge A. Flores Ochoa, una figura fundamental en la antropología cuzqueña y peruana por sus múltiples méritos como investigador, docente, intelectual comprometido y animador cultural infatigable. El doctor Flores Ochoa obtuvo en 1960 un bachillerato en Derecho, en 1965 su licenciatura en Antropología y en 1967 su doctorado en Letras y Ciencias Humanas, grados y títulos conferidos por la Universidad Nacional de San Antonio Abad del $\mathrm{Cuzco}^{1}$. Su alma mater sería también donde ejercería por largos años la investigación y la docencia universitaria.

A lo largo de su carrera, el doctor Flores Ochoa fue profesor visitante de universidades en Japón, Alemania, Suiza, España, Bolivia, Chile, México y Estados Unidos. Ganó prestigiosas becas de investigación académica, entre ellas la de la Fundación Ford (1968) y la de la Fundación Guggenheim (1977). Como parte de sus labores universitarias, fue también director del Museo Inka y ocupó diversos cargos administrativos y de autoridad, entre ellos el rectorado de la UNSAAC. Fuera del claustro universitario se desempeñó como director de Instituto Nacional de Cultural del Cuzco (1980). A lo largo de su trayectoria obtuvo numerosas distinciones y reconocimientos por sus contribuciones al conocimiento de las sociedades andinas, entre ellas ser profesor honorario de la Universidad Nacional Mayor de San Marcos (2003), un homenaje a la trayectoria en el IV Congreso Nacional de Investigación Antropológica (2005) y la Orden al Mérito por Servicios Distinguidos en el Grado de Gran Cruz, la máxima distinción que otorga el Estado peruano (2011)2.

Sin duda un eje innovador, sostenido y muy fructífero del trabajo del doctor Flores Ochoa ha sido el estudio de las sociedades de pastores de camélidos sudamericanos en las punas de Puno y Cuzco. Este se inauguró con su tesis de

\footnotetext{
En este texto escribo Cuzco y cuzqueño siguiendo la forma en que el doctor Flores Ochoa prefirió hacerlo en todos sus textos.

2 Estos datos biográficos tienen como fuente el texto de Hernández (2004).
} 
doctorado que fue su primer libro: Pastores de Paratía, una introducción a su estudio (Flores Ochoa, 1968), que fue posteriormente traducido al inglés como Pastoralists of the Andes: the alpaca herders of Paratia (Flores Ochoa, 1979). Este estudio es innegablemente un hito en la antropología andina. Hasta su publicación no había una etnografía focalizada en alguna sociedad de pastores en los Andes y se asumía que todas las sociedades andinas combinaban el pastoreo con la agricultura, esta última como actividad preponderante. La etnografía de los pastores de Paratía, una comunidad quechuahablante de Lampa, Puno, mostró el complejo mundo social implicado en el manejo de las alpacas, las formas de clasificación de estos animales, las formas de organización de parentesco de los pastores y las prácticas rituales asociadas al manejo y reproducción de las alpacas. A lo largo de toda su carrera, el doctor Flores Ochoa permaneció siempre muy interesado en este tema y siguió contribuyendo con publicaciones sobre múltiples aspectos del pastoreo altoandino.

Los temas cubiertos por sus artículos relacionados al pastoreo de camélidos son muy variados: desde el análisis de la Clasificación y nominación de camélidos sudamericanos (Flores Ochoa, 1981) a la actuación política de los pastores, como en La protesta de 1909: un movimiento de pastores de la puna alta a comienzos del siglo XX (Flores Ochoa y Palacios Ríos, 1978) o su análisis de las prácticas de relacionamiento con el paisaje en tanto este último emerge como una serie de entidades animadas. Un clásico de esto último es el artículo Enqa, enqaychu, illa y khuya rumi: Aspectos mágico-religiosos entre pastores en el que analiza las prácticas alrededor de las miniaturas de piedra que median las relaciones entre los seres del paisaje y la comunidad de seres humanos (Flores Ochoa, 1974). Otro artículo que discute temas relacionados es el muy posterior La Missa Andina (Flores Ochoa, 1998). Si bien enmarca estos temas en las categorías de religión y magia, sus descripciones y análisis muestran lógicas prácticas que claramente las exceden. Cercanos a esta temática se encuentran artículos que discuten formas en las que prácticas y narrativas referidas a estos seres están claramente imbricadas con procesos socioculturales contemporáneos, mostrando que están lejos de ser simples rezagos de un pasado condenado a desaparecer (Flores Ochoa, 1971, 1973). A estas múltiples facetas de sus contribuciones sobre los pastores de puna debemos añadir sus esfuerzos por brindar balances de la investigación realizada sobre estos temas, como en el artículo Pastoreo de llamas y alpacas en los Andes: balance bibliográfico (Flores Ochoa, 1983b) muestra.

$\mathrm{Su}$ rol en la investigación de las sociedades de pastores fue también el de organizador, animador y coordinador de investigaciones de muchos otros autores, 
creando contextos y publicaciones que ponían en diálogo una creciente literatura especializada. Los libros editados por él a lo largo de décadas, como Pastores de puna. Uywamichiq punarunakuna (Flores Ochoa, 1977), Llamichos y paqocheros: pastores de llamas y alpacas (Flores Ochoa, 1988), Oro de los Andes: Las llamas, alpacas, vicuñas y guanacos de Sudamérica (Flores Ochoa, 1994), Pastoreo Altoandino: realidad, sacralidad y posibilidades (Flores Ochoa y Kobayashi, 2000), muestran este esfuerzo sostenido de entablar diálogos y relaciones entre las distintas áreas de conocimiento del pastoreo de puna.

Su reflexión sobre estas sociedades no se limitó a su descripción etnográfica y trabajo histórico y etnohistórico, sino también incluyó esfuerzos por mostrar las potencialidades económicas y sociales de las sociedades de pastores y de los recursos de las punas. Criticando las miradas estatales que tienden a reducir a estas poblaciones a índices de pobreza y a población dispersa que hay que concentrar, él argumentó incesantemente que una comprensión más cercana y empática de sus formas de organización, lógicas económicas y de manejo espacial podría brindar luces y caminos de mejora en las condiciones de vida y de bienestar de estas comunidades. No se cansó de criticar la inacción y miopía estatal frente a la potencialidad de los camélidos como fuentes de riqueza, trabajo y bienestar.

Un tema cercano a sus investigaciones sobre el pastoreo fue su interés en el manejo de la complejidad ecológica de los Andes. Este interés está quizás asociado a su temprana relación con John V. Murra (Flores Ochoa, 2010). Su artículo El reino de Lupaqa y el actual control vertical de la ecología (Flores Ochoa, 1972) no solo está en diálogo con el texto de Murra (1972) sobre el archipiélago vertical, sino que discute aspectos de la visita de Garci Diez de San Miguel en relación con el presente etnográfico. Otro de sus textos sobre esta temática va más allá del pastoreo. En La agricultura en lagunas del altiplano Flores Ochoa (1983a) analiza las tecnologías de manejo de tierras inundables mediante la tecnología de los waru waru que hacen posible la agricultura intensiva en la puna. Este interés en la adaptación de las poblaciones andinas a la complejidad ecológica es en el núcleo del libro Puna, qheswa, yunga: el hombre y su medio en Q'ero, en coautoría con Ana María Fríes. En él explica el manejo vertical de tres pisos ecológicos por los comuneros de Hatun Q'ero (Paucartambo, Cuzco) que se lleva a cabo en el ámbito familiar y que implica una serie de cambios de residencia a diferentes altitudes a lo largo del ciclo agrícola anual (Flores Ochoa y Fries, 1989).

Relacionado con esta comunidad, también fue editor, junto con Juan Víctor Núñez del Prado, del libro Q'ero, el último ayllu inka: homenaje a Óscar Núñez del Prado Castro (Flores Ochoa y Núñez del Prado, 1983). Este reúne los artícu- 
los que fueron escritos, y la mayoría publicados previamente, por los diferentes investigadores de la UNSAAC que viajaron conjuntamente a la entonces hacienda Q'ero en 1955 frente a la oposición de hacendados, la prefectura, e incluso las autoridades universitarias. Para él, tal como sostiene enfáticamente en ¿Por qué Q'ero?, la introducción al libro, estas investigaciones fueron fundamentales en moldear el espíritu del programa de antropología de la UNSAAC. Flores Ochoa enfatiza la interdisciplinariedad que emergió de la combinación de los distintos investigadores involucrados y las contribuciones pioneras a debates claves en la antropología andina en los años por venir, entre ellos el registro de la primera versión del mito de Inkari, la verticalidad agroecológica andina y la evidencia etnográfica del parentesco bilateral andino. Asimismo, subraya una apuesta no solo por la investigación sólidamente anclada en la etnografía sino también por la antropología aplicada.

Otro aspecto claramente presente en su obra es su interés, no solo en la etnohistoria sino en la cultura material y el arte colonial cuzqueño. Varios de estos trabajos los publicó conjuntamente con la antropóloga e historiadora del arte Elizabeth Kuon Arce y el arquitecto Roberto Samanez Argumedo. Los temas que abordó incluyen la pintura mural, tanto religiosa como secular, en los libros Pintura Mural en el Sur Andino (Flores Ochoa et al., 1993) y Pintura mural cuzqueña del siglo XX (Flores Ochoa et al., 1992a); el análisis de los vasos inka de madera, en especial aquellos polícromos coloniales y su rol en la reproducción de ideologías incanistas en la colonia (Flores Ochoa, 1990a; Flores Ochoa et al., 1998); la arquitectura colonial urbana y los retos de su restauración, como en el libro La Casa de la Calle Tigre, la casona colonial que actualmente alberga al Rectorado de la UNSAAC (Samanez Argumedo et al., 1992). Este acercamiento al análisis de las artes coloniales estuvo asociado a un creciente interés en la historia y la etnografía de la ciudad del Cuzco desde mediados de la década de 1980.

Su interés en la larga historia de la ciudad y en sus manifestaciones culturales contemporáneas estuvo muy relacionado con su convicción de analizar al Cuzco en tanto cuzqueño, y esto implicó para él analizar y al mismo tiempo abrazar el incanismo en el que todos los cuzqueños, en distintas formas, hemos crecido (Flores Ochoa, 2005a). Muchas de sus publicaciones sobre la ciudad del Cuzco están relacionadas con pensar este incanismo, este «sentimiento de identidad con lo inca, con las glorias reales o supuestas del Tawantinsuyo», en clave de resistencia a presiones e imposiciones culturales y subrayando la continuidad de formas culturales prehispánicas y coloniales que pueden ser encontradas en el presente (Flores Ochoa, 1990b, p. 11). Estas resistencias pueden ser pasivas, 
inscritas en las decoraciones de los vasos qero, o tremendamente violentas, como en las múltiples rebeliones anticoloniales del s. XVIII y temprano s. XIX.

Para Flores Ochoa, la idea del Inka es un mito fundamental de la ciudad y de la región del Cuzco. El rastreo de la idea del Inka asociada a lo que llama incanismo se encuentra en su lectura de las crónicas tempranas a través de las que se puede tener un acercamiento de lo que llama los incas históricos para también rastrear sobre la larga tradición de la construcción de la idea del Inka inscrita en el incanismo, ubicando al Inca Garcilaso de la Vega como su fundador (Flores Ochoa, 1990b), continuando con la emergencia de lo que Rowe (1954) llamó el Movimiento Nacional Inka del s XVIII y desembocar en el Indigenismo Cuzqueño de inicios del s XX. Es importante señalar que Flores Ochoa reclamó al Indigenismo Cuzqueño como una influencia fundamental de la escuela de antropología de la UNSAAC (Flores Ochoa, 1983c).

La creciente omnipresencia de la idea del Inka en la vida de la ciudad y cada vez más de la región está claramente inscrita en la festividad del Inti Raymi que Flores Ochoa muestra cómo, más allá de la usual afirmación de que solo consiste en un espectáculo para turistas, esta ha sido cada vez más apropiada por los heterogéneos habitantes de la ciudad y replicada en innumerables festividades inspiradas en este en muchas zonas de la región y del país (Flores Ochoa, 2005b). En un argumento sugerente y polémico que vincula su antropología de la ciudad con su conocimiento de las prácticas de pastores de puna, Flores Ochoa equipara y vincula la idea del Inka como héroe fundamental de la mitología cuzqueña con el enqa, un principio generador y vital, fuente del bienestar y la abundancia. Propone así una multiplicidad articulada de formas de entender al Inka que consolidan y retroalimentan, a pesar de sus diferencias, en su centralidad de articulación de ideologías cuzqueñas contemporáneas (Flores Ochoa, 2002).

Profundamente asociado a estos temas, un conjunto de trabajos de Flores Ochoa está dedicado al análisis y reflexión sobre las celebraciones religiosas de la ciudad. Entre estas no podía faltar la procesión celebratoria cuzqueña por excelencia, el Corpus Christi, a la que se refiere como «la fiesta de los cuzqueños» (Flores Ochoa, 1990c) y el culto al Señor de los Temblores y los muchos otros más que se llevan a cabo en catedral (Flores Ochoa, 2013). Su visión de la complejidad de estas celebraciones se puede encontrar en el volumen, en coautoría con Elizabeth Kuon Arce y Roberto Samanez Argumedo, Cuzco, el lenguaje de la fiesta (Flores Ochoa et al., 2009). Otro aspecto asociado profundamente a la ciudad fue su interés en los procesos asociados a la creciente importancia del turismo en el Cuzco, su relación con la etnicidad y el incanismo. Fue particularmente 
crítico del llamado turismo místico en particular sobre los usos superficiales e impostados de prácticas indígenas con fines comerciales (Flores Ochoa, 1996; van den Berghe y Flores Ochoa, 2000).

Su interés por la antropología de la ciudad lo llevó a promover investigaciones y publicaciones de múltiples autores que cubrieron una variedad de estudios, tanto en la ciudad como en zonas circundantes, y que incluyen diversos aspectos como celebraciones de santos patronos y vírgenes, nuevas prácticas asociadas a la migración puneña en el Cuzco, aquellas asociadas al culto a las cruces, el despacho urbano y las prácticas de los sorprendentemente numerosos curanderos urbanos. Frutos de estos esfuerzos son las compilaciones Qosqo. Antropología de la ciudad (Tomoeda y Flores Ochoa, 1992) y Celebrando la fe: fiesta y devoción en el Cuzco (Flores Ochoa, 2009).

Un texto que vincula este interés por las celebraciones religiosas y su extenso conocimiento sobre sociedades pastoras de puna es su análisis de la peregrinación de Qoyllurit'i. Su perspectiva, como podría esperarse, es una visión de esta peregrinación desde las prácticas de los pastores. Él es categórico en subrayar que esta peregrinación está profundamente asociada a las prácticas de pastores de puna. En particular subraya que los pawluchas son alpacas y no osos, como usualmente se sostiene, en asociación con su otro nombre, ukuku. Si bien esto último es debatible, es claramente muy certero en su discusión sobre el nombre de la peregrinación, su escritura y su significado. La escritura del nombre de la peregrinación se estuvo haciendo de muchas variadas formas, con Qoyllur Rit'i como la más extendida, a la que se suele traducir como estrella de nieve. Es claro que esta traducción es inexacta, y una más cercana al quechua tendría que ser algo así como nieve estelar. Flores Ochoa argumenta que esa forma de escritura no corresponde con la pronunciación extendida de esta palabra que es más bien Qoyllurit'i. Qoyllu se refiere a un tipo de blanco de ciertas lanas de alpaca, un blanco resplandeciente, de modo que Qoyllurit'i más bien corresponde con nieve resplandeciente (Flores Ochoa, 1990d).

Algo que muestra su rol como intelectual público y animador cultural fue su intervención respecto del nombre de la peregrinación que rebasó un plano puramente académico. El asistió a la presentación de un documental realizado por la Hermandad del Señor de Qoyllurit'i (que no escribía entonces el nombre de esta manera) sobre la peregrinación y en esta comentó críticamente el asunto del nombre y su escritura. Si bien inicialmente esto provocó reacciones defensivas de los representantes de la hermandad, el asunto desencadenó un diálogo fructífero con la hermandad, de modo que un tiempo después esta asumió como 
suya la posición del doctor Flores Ochoa. Actualmente la Hermandad, el Consejo de Naciones Peregrinas, así como el Ministerio de Cultura, escriben el nombre de la peregrinación según su propuesta y sus razones.

Alguien relativamente familiarizado notará que este repaso por sus temas de interés más importantes no supone una revisión exhaustiva de todas sus investigaciones. Más allá de esto, vale la pena mencionar que, significativamente, los dos últimos libros que lideró estuvieron dedicados a las dos regiones a las que dedicó el grueso de sus esfuerzos intelectuales y con las que estaba profundamente involucrado: Cuzco: desde la nieve de la puna al verdor de la Amazonía (Flores Ochoa et al., 2011) y La magia del agua en el lago Titicaca (Flores Ochoa y Gisbert, 2012).

Su vasta obra, sin embargo, no solo se encuentra en sus numerosos libros y artículos, sino también en las relaciones con estudiantes, colegas y ciudadanía en general, en sus múltiples intervenciones en la esfera pública, siempre acompañado de una sobria elegancia, una actitud respetuosa y su don de gentes. Fue un gran animador y corazón de la antropología cuzqueña por largos años. Fue, junto con Luis Barreda Murillo y Abraham Valencia, uno de los motores del Tinkuy Anual del Centro de Estudios Andinos Cuzco. Este evento se constituyó en un lugar de encuentro donde se presenta el grueso de investigación antropológica en curso en la región. Su programa de radio, del mismo nombre y del mismo colectivo, fue también una de las formas en las que participaba activamente en la vida cultural de ciudad, más allá de sus conferencias y publicaciones.

Fue durante mi trabajo de campo doctoral en 2008 y 2009 cuando lo pude conocer más allá de la lectura de sus textos. Me encontré con él muchas veces, no solo en sus charlas en el Qorikancha o en el Museo Inka, sino sobre todo cuando estaba haciendo lo mismo que él hacía: observando, conversando, fotografiando, tomando notas. Recuerdo haberlo encontrado en Qoyllurit'i en un momento en que él estaba registrando cómo equipos de grabación de video abusaban de la hospitalidad de los peregrinos, entrometiendo sus cámaras sin consideración ni respeto y perturbando las danzas de las comparsas; en la fiesta de San Jerónimo, sobre la que me comentó cómo, de ser una fiesta bastante pequeña cuando la conoció en su juventud, se había transformado en la explosión barroca que estábamos observando; también en la expresión más impresionante del barroco cuzqueño contemporáneo, el Corpus Christi; en los concursos de danza por las fiestas del Cuzco comentando cómo sería imposible encontrar mucho de lo que veíamos en las comunidades que el presentador oficial mencionaba como su lugar de origen; observando y escuchando los himnos de las ch'ayñas del Señor del Temblores 
o alegre acompañando a una tropa de sikuris en un anochecer en la Plaza de Armas. Seguramente, cuando este texto esté publicado, él ya habrá llegado a su nueva morada, allá hacia al oeste, en la tierra de los volcanes, donde — qué duda cabe - seguirá conversando, tomando notas y escribiendo sobre todo aquello a lo que ha dedicado sus esfuerzos en este mundo.

\section{REFERENCIAS}

Flores Ochoa, Jorge A. (1968). Los pastores de Paratía, una introducción a su estudio. México: Instituto Indigenista Interamericano.

Flores Ochoa, Jorge A. (1971). La wak'a awicha Anselma. Allpanchis Puthurinqa, 3, 68-78.

Flores Ochoa, Jorge A. (1972). El reino de Lupaqa y el actual control vertical de la ecología. Historia y Cultura, 6, 195-202.

Flores Ochoa, Jorge A. (1973). La viuda y el hijo del Soq'a Machu. Allpanchis Puthurinqa, 5, 45-55.

Flores Ochoa, Jorge A. (1974). Enqa, enqaychu, illa y khuya rumi: Aspectos mágicoreligiosos entre pastores. Journal de la Société des Américanistes, 63, 245-262.

Flores Ochoa, Jorge A. (ed.). (1977). Pastores de puna. Uywamichiq punarunakuna. Lima: Instituto de Estudios Peruanos.

Flores Ochoa, Jorge A. (1979). Pastoralists of the Andes: The alpaca herders of Paratia. Filadelfia: Institute for the Study of Human Issues.

Flores Ochoa, Jorge A. (1981). Clasificación y nominación de camélidos sudamericanos. En Heather Lechtman y Ana María Soldi (eds.), La tecnología en el mundo andino. México: Universidad Nacional Autónoma de México.

Flores Ochoa, Jorge A. (1983a). La agricultura en lagunas del altiplano. Ñawpa Pacha, 21(1), 127-152.

Flores Ochoa, Jorge A. (1983b). Pastoreo de llamas y alpacas en los Andes: Balance bibliográfico. Revista Andina, 1(1), 175-218.

Flores Ochoa, Jorge A. (1983c). ¿Por qué Q'ero? En Jorge A. Flores Ochoa y J. V. Núñez del Prado (eds.), Q'ero, el último ayllu inka: Homenaje a Oscar Núñez del Prado Castro. Cuzco: Centro de Estudios Andinos Cuzco.

Flores Ochoa, Jorge A. (ed.). (1988). Llamichos y paqocheros. Pastores de llamas y alpacas. Cuzco: CEAC, CONCYTEC. 
Flores Ochoa, Jorge A. (1990a). Arte de resistencia en vasos ceremoniales Inka. Siglos XVII-XVIII. En El Cvzco. Resistencia y continuidad (pp. 15-72). Cuzco: Centro de Estudios Andinos Cuzco.

Flores Ochoa, Jorge A. (1990b). Inkanismo, resistencia, y continuidad cuzqueños. En El Cvzco. Resistencia y continuidad (pp. 9-14). Cuzco: Centro de Estudios Andinos Cuzco.

Flores Ochoa, Jorge A. (1990c). La fiesta de los cuzqueños: La procesión del Corpus Christi. En El Cvzco. Resistencia y continuidad (pp. 9-14). Cuzco: Centro de Estudios Andinos Cuzco.

Flores Ochoa, Jorge A. (1990d). Taytacha Qoyllurit'i. El Cristo de la Nieve Resplandeciente. En El Cvzco. Resistencia y continuidad (pp. 73-94). Cuzco: Centro de Estudios Andinos Cuzco.

Flores Ochoa, Jorge A. (ed.). (1994). Oro de los Andes: las llamas, alpacas, vicuñas y guanacos de Sudamérica. Barcelona: F. O. Patthey e hijos.

Flores Ochoa, Jorge A. (1996). Buscando los espíritus de Ande. Turismo místico en el Qosqo. En Hiroyasu Tomoeda y Luis Millones (eds.), La tradición andina en tiempos modernos (pp. 9-29). Osaka: Museo Nacional de Etnología de Japón.

Flores Ochoa, J. A. (1998). La missa andina. En Actas del IV Congreso Internacional de Ethnohistoria (pp. 99-115). Lima: Fondo Editorial PUCP.

Flores Ochoa, Jorge A. (2002). El Inka histórico es el Enqa contemporáneo. En Javier Flores Espinoza y Rafael Varón Gabai (eds.), El hombre y los Andes: Homenaje a Franklin Pease G.Y. (Vol. 2, pp. 611-616). Lima: Fondo Editorial PUCP.

Flores Ochoa, Jorge A. (2005a). El Centro del Universo Andino. Cuzco: Municipalidad del Cusco.

Flores Ochoa, J. A. (2005b). «En el principio fue el Inka». El ciclo del Inti Raymi cuzqueño. En Nueva Antología del Cusco (pp. 278-299). Cuzco: Municipalidad del Cusco.

Flores Ochoa, Jorge A. (ed.). (2009). Celebrando la fe: Fiesta y devoción en el Cuzco. Cuzco: UNSAAC, CBC.

Flores Ochoa, Jorge A. (2010). John Victor y los «andinos». Chungará, 42(1), 25-28. Recuperado de http://www.jstor.org/stable/23050869

Flores Ochoa, Jorge A. (2013). El culto popular. En Tesoros de la Catedral del Cusco (pp. 18-44). Cuzco: Arzobispado de Cusco, Dirección Desconcentrada de Cultura de Cusco, Telefónica del Perú.

Flores Ochoa, Jorge A. y Ana María Fries (1989). Puna, Qheswa, Yunga: El hombre y su medio en Q'ero. Lima: Fondo Editorial del Banco Central de Reserva del Perú. 
Flores Ochoa, J. A. y Teresa Gisbert (eds.). (2012). La magia del Agua en el lago Titicaca. Lima: Banco de Crédito del Perú.

Flores Ochoa, J. A. y Yoshiki Kobayashi (eds.) (2000). Pastoreo altoandino: realidad, sacralidad y posibilidades. La Paz: Plural.

Flores Ochoa, J. A., Elizabeth Kuon Arce y Roberto Samanez Argumedo (1992a). Pintura mural cuzqueña del siglo XX. UNSAAC.

Flores Ochoa, Jorge A., Elizabeth Kuon Arce y Roberto Samanez Argumedo (1993). Pintura mural en el sur andino. Lima: Banco de Crédito del Perú.

Flores Ochoa, Jorge A., Elizabeth Kuon Arce y Roberto Samanez Argumedo (1998). Qeros: Arte Inka en vasos ceremoniales. Lima: Banco de Crédito del Perú.

Flores Ochoa, Jorge A., Elizabeth Kuon Arce y Roberto Samanez Argumedo (2009). Cuzco, el lenguaje de la fiesta. Lima: Banco de Crédito del Perú.

Flores Ochoa, Jorge A., Elizabeth Kuon Arce y Roberto Samanez Argumedo y Donato Amado González (2011). Cuzco: desde la nieve de la puna al verdor de la Amazonía. Lima: Banco de Crédito del Perú.

Flores Ochoa, Jorge A. y Juan V. Núñez del Prado (1983). Q’ero, el último ayllu inka: Homenaje a Oscar Núñez del Prado Castro. Cuzco: Centro de Estudios Andinos Cuzco.

Flores Ochoa, Jorge A. y Félix Palacios Ríos (1978). La protesta de 1909. Un movimiento de pastores de la puna alta a comienzos del Siglo XX. Debates en Antropología, 2, 75-88.

Hernández, Harold (2004). Jorge Flores Ochoa: Profesor Honorario de la UNMSM. Revista de Antropología, 2(2), 79-85.

Murra, John V. (1972). El control vertical de un máximo de pisos ecológicos en la economía de las sociedades andinas. En Visita de la Provincia de León de Huánuco en 1562 (pp. 383-406). Huánuco: Universidad Nacional Hermilio Valdizán.

Rowe, John (1954). El Movimiento Nacional Inca del siglo XVIII. Revista Universitaria, 107, 17-47.

Samanez Argumedo, Roberto, Jorge A. Flores Ochoa y Elizabeth Kuon Arce (1992). La Casa de la Calle Tigre: recuperación y puesta en valor de una casa del Cuzco. Cuzco: UNSAAC.

Tomoeda, Hiroyasu y Jorge A. Flores Ochoa (1992). El Qosqo: antropología de la ciudad. Cuzco: Ministerio de Educación del Japón, Centro de Estudios Andinos Cuzco.

van den Berghe, Pierre L. y Jorge A. Flores Ochoa (2000). Tourism and nativistic ideology in Cuzco, Peru. Annals of Tourism Research, 27, 7-26. 